\title{
QoS-Aware Middleware for Ubiquitous and Heterogeneous Environments
}

\author{
Klara Nahrstedt, University of Illinois at Urbana-Champaign \\ Dongyan $\mathrm{Xu}$, Purdue University \\ Duangdao Wichadakul, University of Illinois at Urbana-Champaign \\ Baochun Li, University of Toronto
}

\begin{abstract}
$M$ iddleware systems have emerged in recent years to support applications in heterogeneous and ubiquitous computing environments. Specifically, future middleware platforms are expected to provide quality of service support, which is required by a new generation of $\mathrm{Q} O \mathrm{~S}$-sensitive applications such as media streaming and e-commerce. This article presents four key aspects of a Q oS-aware middleware system: Q oS specification to allow description of application behavior and Q oS parameters; Q oS translation and compilation to translate specified application behavior into candidate application configurations for different resource conditions; Q OS setup to appropriately select and instantiate a particular configuration; and finally, Q OS adaptation to adapt to runtime resource fluctuations. W e also provide a comparison of existing Q oS-aware middleware systems in these four aspects.
\end{abstract}

\section{INTRODUCTION}

A new generation of distributed applications, such as telemedicine and e-commerce applications, are being deployed in heterogeneous and ubiquitous computing environments. These applications are expected to deliver adaptive and satisfactory quality of service (Q $0 S)$, in order to be accepted by general users. This poses a challenge in the support of Q OS specification, setup, and enforcement for these applications.

In the past decade, various architectures, protocols, and algorithms have been proposed to address these challenging issues. For example, solutions have been proposed for setting up and enforcing Q OS in IP or asynchronous transfer mode (ATM) networks, in operating system (OS) kernels, and in applications themselves. While network and OS-level solutions provide native and generic Q oS support, they may not be easily and rapidly deployed on a large scale and for all new applications. On the other hand, application-level solutions, such as adaptive or layered video coding, may be applicable only to a certain application domain.

$M$ ore recently, various solutions at the middleware layer have also been presented, which reside between applications and OS kernels. In comparison, middleware solutions provide more flexibility when assisting new applications in ubiquitous computing environments. In this article we propose our solution to Q oS specification, setup, and enforcement at the middleware layer. 0 ur middleware easily cooperates with existing solutions at OS, network, and application levels. Furthermore, even when OS or networks are best effort rather than Q oS-enabled, the middleware system can still assist applications with Q OS adaptations. O ur solution spans from Q OS specification and translation in the development phase of an application to QoS setup and adaptation at runtime. We believe that these capabilities are essential to any Q oSaware middleware system.

The remainder of this article is organized as follows. We will present an architectural overview of our Q OS-aware middleware and discuss Q OS specification and compilation issues. Then we will present Q oS setup and adaptation approaches, compare existing middleware solutions in related work, and conclude with lessons learned.

\section{QOS-AWARE \\ MIDDLEWARE ARCHITECTURE}

O ur Q oS-aware middleware architecture favors applications modeled by a generic application component model. In this model we view a collection of interconnected application components 


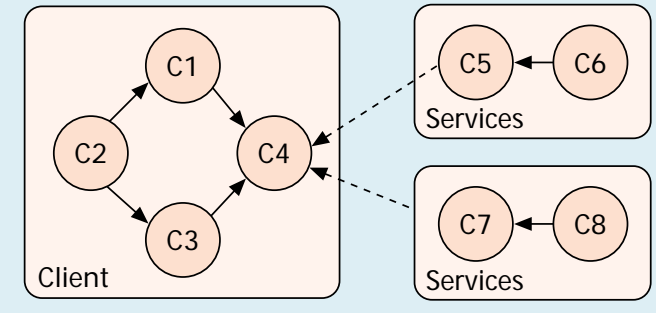

(a) An application functional graph

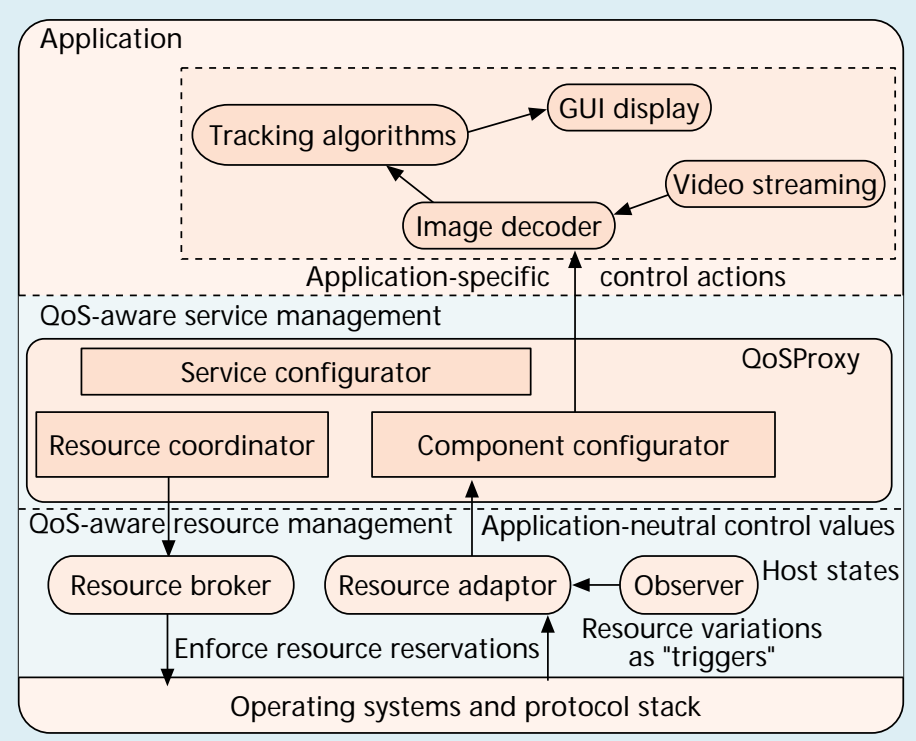

(b) Components in a QoS-aware middleware architecture

Figure 1. Q oS-aware middleware architecture: an overview.

on a single host as a set of tasks, with input-output dependencies. B eyond a single end host, we group the entire distributed application into clients and services. The collection of clients and services form another directed graph representing the service provider-consumer relations. This graph is called an application functional graph, as illustrated in Fig. 1a.

In fact, our Q oS-aware middleware is a component base system itself. I ts architecture is shown in Fig. 1b, with components at both Q oSaware resource management and $\mathrm{Q}$ oS-aware service management levels. A $n$ instance of this architecture is running in every end host in the environment:

- QoS-aware resource management consists of resource brokers, resource adaptors, and observers. They are responsible for resource reservation, enforcement, adaptation, and monitoring. Q oS-aware resource management is built on top of individual $O S$ and network resource management functions, such as the reservation and scheduling of CPU, disk, and network bandwidth.

- QoS-aware service management is represented by a collection of middleware components, collectively referred to as the Q oSProxy. The decisions and actions of a Q oSProxy are driven by resource conditions reported by the underlying resource management components. The definitions of these decisions and actions are initially injected via Q oS specification and compilation (to be described in the next section), and they reflect the middleware's capabilities of service discovery, application configuration selection/reselection/instantiation, and coordinated multiresource allocation. It is worth noting that Q oSProxies operate only on the control/management plane of an application, not on its data plane. Therefore, they do not hinder the processing and transmission of the application data.

For an application, the Q oS-aware middleware provides support spanning from its development phase to its runtime phase:

- During the development phase (see the next section), the application developer specifies Q oS parameters, possible configurations, and applicable environments of an application. The specifications are then translated by the Q OS compiler, a companion development tool of the middleware (not shown in Fig. 1b), into internal representations, which will be injected into the middleware and used at runtime.

- During the runtime phase, the QoS-aware middleware performs Q OS setup and adaptation for the application. Q oS setup (discussed later) takes place right before the execution of the application, while Q OS adaptation (also discussed later) is triggered during the application execution by resource fluctuation, user mobility, and change of user preference.

\section{Application DeVelopment Phase \\ QOS SPECIFICATION}

During the application development phase, an application developer provides QoS specification about the target application. The format of Q OS specification varies in different Q oS-aware middleware systems. F or example, in Q oSM E [1], Q oS is described via a Q uality of Service A ssurance $L$ anguage ( $Q U A L$ ); in A gilos [2], Q o $S$ is defined via rules and membership functions; while in Q-R A M [3], Q oS is represented by resource utility functions. H owever, Q oS specifications share the following characteristics:

- They are application-specific.

- Their formats are tailored for the targeted application domains.

- They need translations from the original 


Major steps in
QoS setup
include service
discovery,
application
configuration
selection, and
resource
allocation. In
addition, if the
user is mobile,
QoS setup also
performs
application-level
handoff when
the user's
location or
physical
environment
changes.

${ }^{1}$ We use these terms in accordance with the Internet E ngineering Task Force (IETF) Service L ocation Protocol specification [5]. application-level notations into the systemlevel Q oS parameters and representations.

F or Q oS specification of applications in ubiquitous environments, we adopt a representation which includes:

- A $n$ application description detailing the set of participating application components, the application Q oS parameters and levels, and the mapping function from user-perceived Q oS levels to the application Q oS levels

- Application adaptation policies indicating when and how the application should adapt to changing environments and resource conditions (to be detailed later)

- A $n$ application state template defining the necessary state information with which the application execution can properly pause and resume

F or example, the application state template of a media streaming application may be specified as its current video and audio frame numbers. 0 ur middleware supports a companion QoS programming environment that helps developers conform to such a Q oS specification format.

\section{QOS COMPILATION}

A fter accepting the Q oS specification of an application, the Q OS compiler translates the specification into a Q OS profile. The Q OS profile serves as both a "contract" and a "script" to be followed by the Q oS-aware middleware at runtime. Q oS compilation is analogous to program compilation: the application source code is translated into an object code by the language compiler so that at runtime the object code will be executed by the runtime support system. Similarly, the QoS profile - the "object code" generated by the QoS compiler - will be "executed" by the Q oS-aware middleware system for the setup, delivery, and adaptation of application Q OS.

W ith Q oS specification as the "source code," Q oS compilation proceeds as follows (more details can be found in [4]):

- Step 1:The QoS compiler translates the Q oS specification into a set of application functional graphs. Each graph contains a different set of application components, representing a possible configuration of the application. In ubiquitous and heterogeneous environments, it is desirable for an application to have multiple configurations, each suited to a different $\mathrm{Q}$ oS requirement, resource condition, or physical environment of users.

- Step 2: The Q oS compiler associates each application functional graph with appropriate system service components - components which perform domain-specific but application-independent functions, such as CPU monitors, buffers, or R eal-Time Transfer Protocol (RTP)-based senders and receivers. Step 2 can be seen as a refinement of the application functional graphs generated in step 1.

- Step 3: The Q OS compiler derives the endto-end resource assignment to application components in each application functional graph (i.e., each application configuration). This is done by either analytical resource calculation or experimental resource probing. O ur Q oS compiler only determines the minimum end-to-end resource assignment for each configuration of the application.

The resultant Q OS profile consists of three parts:

- Candidate application configurations and their resource assignments

- Application adaptation policies

- Application state template

\section{Runtime Phase: QoS Setup}

A fter Q oS compilation, the application is ready for deployment: the application components will be installed in the servers or clients of this application; the Q oS-aware middleware runs in each host in the environment; and the result of Q OS compilation - the QoS profile - will first be stored in the Q oSProxy of the application server. A t runtime, parts of the Q OS profile will be downloaded to the Q oSProxy of each client, as will be described shortly.

In this section we present the runtime Q oS setup performed by the Q oS-aware middleware. Q oS setup begins when a user starts an application with a certain $\mathrm{Q} O S$ requirement, and ends when the application begins to execute. The entities involved in this phase are shown in $\mathrm{Fig}$. 2. During Q oS setup, the middleware customizes the application by selecting one of the prespecified configurations. The selection is driven by the user's Q oS requirement and current end-toend resource condition.

\section{QOS Setup Protocol}

$M$ ajor steps in Q oS setup include service discovery, application configuration selection, and resource allocation. In addition, if the user is mobile, Q oS setup also performs applicationlevel handoff when the user's location or physical environment changes.

- Step 1 (Service Discovery): The user's request does not have to designate the location of the corresponding application server. Instead, the user specifies a descriptive service query, including the $\mathrm{Q}$ oS requirement. The query will then be submitted to a Service Discovery System, which is a public infrastructural service (like the DNS) responsible for discovering the server of an application. From a user's point of view, the Service D iscovery System accepts a service query, and returns service handles of a set of qualified servers. Looking into the Service D iscovery System, it consists of three types of entities: user agent ( $U A)$, directory agent (DA), and service agent (SA ). ${ }^{1}$ A s part of the Q OSProxies, the U A S and SA s run in clients and in servers, respectively. The DA s are logically independent brokers between UAs and SA S. A U A intercepts a user's service query and submits it to the DA. M eanwhile, an SA sends a service handle on behalf of the server to the $D A$. U pon receiving a service query from a $U A$, the $D A$ pulls out every qualified service handle that satisfies the query. If there are multiple qualified service handles, either the DA or the UA will make a choice among them. Examples of Service D iscovery Systems include the IE TF Service Location Protocol (SLP) [5], J ini by Sun M icrosystems [6], and 


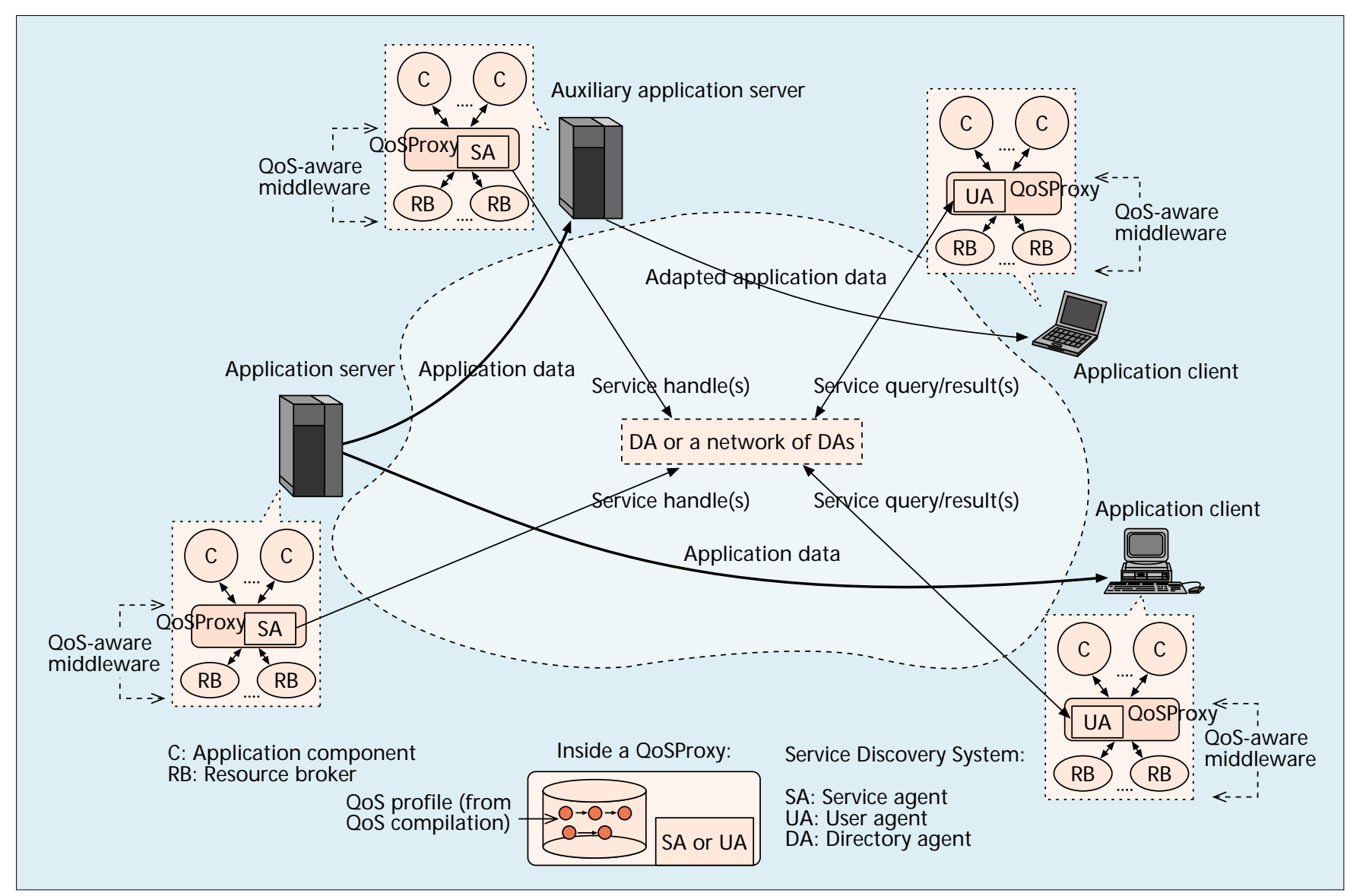

Figure 2. Entities involved in runtime Q oS setup.

Berkeley's Service D iscovery Service (SD S) [7].

Q oS awareness is also an important requirement for a Service D iscovery System. It requires that a discovered server be able to deliver satisfactory Q oS to the querying client. To make a Service D iscovery System Q oS-aware, there exist both server-based and client-based approaches: the former involves the reporting of current server performance status by the SA to the DA; the latter leverages the client feedbacks about recently perceived application Q OS from the U A back to the DA [8]. The DA will then use the server report or Q oS feedback to make Q oS-aware server selections for upcoming service queries.

- Step 2 (Application C onfiguration Selection): A fter the application server has been discovered, the next step is to customize, or configure, the application. In ubiquitous environments, the end-to-end resource conditions observed by clients are highly heterogeneous. For example, the server load or end-to-end network bandwidth may fluctuate, and different clients may have different processing capabilities. Therefore, it is desirable that a ubiquitous application does not execute in a single form. Instead, different configurations will be selected dynamically under different end-to-end resource conditions. A pplication configuration selection is based on both the user Q OS requirement and the Q OS profile generated during QoS compilation. First, the current end-to-end resource condition is collected by querying the resource brokers (RBS) in the client and the server. Sec- ond, the server-side Q oSProxy compares the current resource condition with the resource assignment of each candidate application configuration in the Q oS profile. The configuration is then selected as the one whose resource assignment is satisfied by the current resource condition, and whose resultant end-to-end $\mathrm{QOS}$ is equal to or better than the $\mathrm{Q} O \mathrm{~S}$ requirement specified by the user. If no candidate configuration is able to deliver the required $\mathrm{Q} 0 \mathrm{~S}$, the user may be notified, and the configuration that delivers the best possible end-to-end Q oS under the current resource condition may be selected.

In a selected application configuration, there may be application components that run on some auxiliary application servers. These components perform Q oS customization under the resource condition that this configuration targets. L ocations of the auxiliary application servers also have to be discovered. This is again performed by querying the Service Discovery System.

- Step 3 (R esource Allocation): A fter the application configuration has been selected, and the location of every participating server discovered, the next step is to make multiresource allocation. First, an end-to-end allocation plan will be generated according to the end-to-end resource assignment given in the $\mathrm{Q} O \mathrm{~S}$ profile. Second, the end-to-end allocation plan will be fragmented and dispatched to the Q oSProxies running on the server (locally), the client, and the auxiliary server(s) - if any. Third, after 


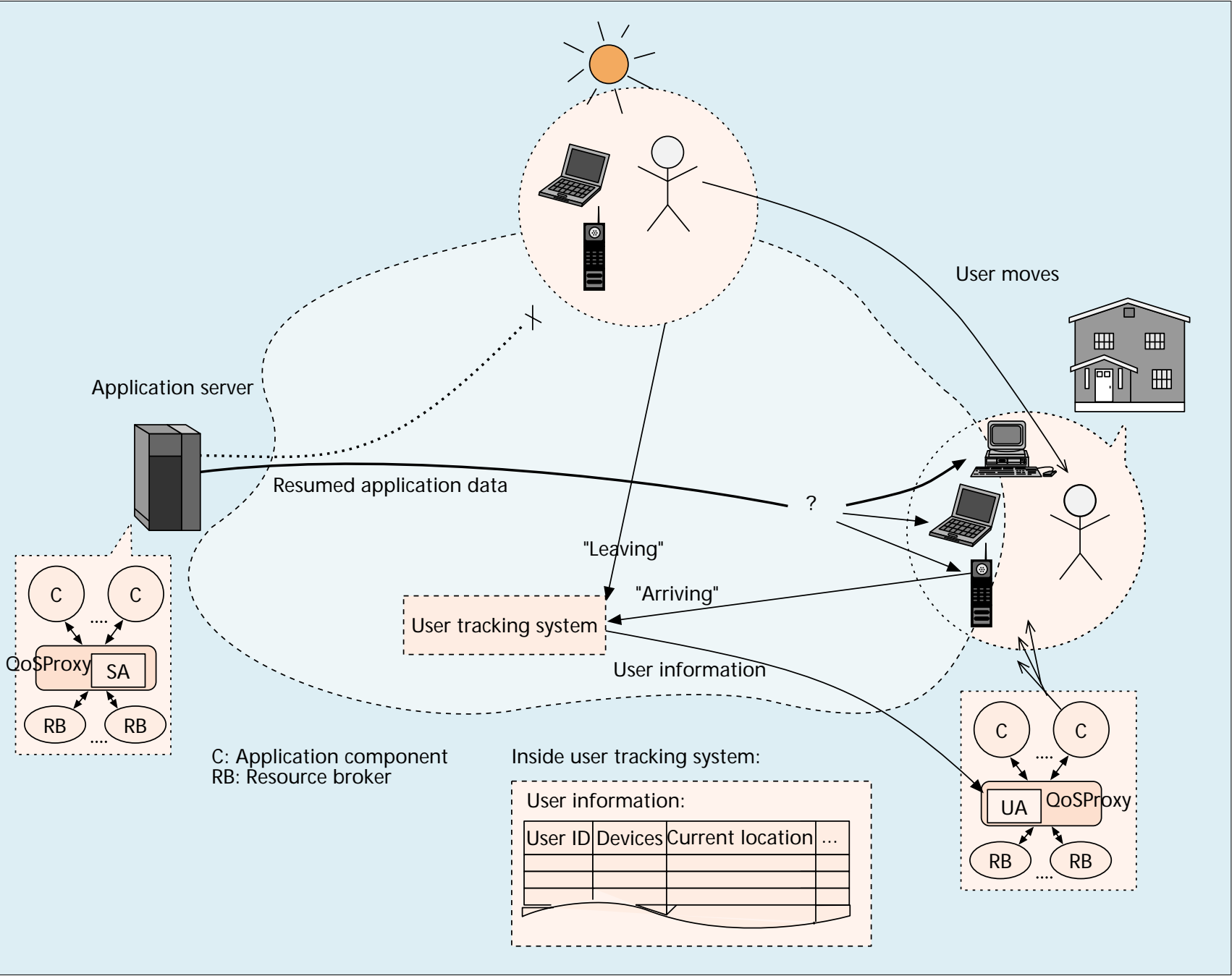

Figure 3 U ser mobility and application-level handoff.

receiving the corresponding segment of the endto-end resource allocation plan, the Q OSProxy running on that host will further dispatch the plan to the local R Bs. Finally, the R Bs will make the actual allocations.

- Step 4 (Q oS Profile Downloading): The client-side Q OSProxy will download the following two parts of the Q OS profile from the server-side Q oSProxy: application adaptation policies and an application state template. Application adaptation policies are for Q oS adaptation (see a later section), while the application state template is for the support of application-level mobility.

When the four steps are completed, the Q oSProxy on each host will start the local application component(s) involved in the selected configuration. The execution of the application will then begin.

\section{ApPLiCATION-LeVel Mobility SUPPORT}

In ubiquitous environments, the mobility of users should be treated as a normal case instead of as an exception. This requires that the $\mathrm{Q}$ oS setup also incorporates support for user mobility, as illustrated in Fig. 3. A user may start an application, and then move to another location. The user may move with the same client machine, with no client machine, or even with multiple client machines such as a laptop computer, PDA, and cellular phone. A the new location, the Q oS setup must accommodate the continuation of the application: its intermediate execution state has to be restored; and its client machine as well as the corresponding application configuration may have to be redetermined. The reason for a possible change of client machine is the user's changing physical environment. For example, a user at home uses a desktop PC to view an online music video. However, when he/she gets into a car, the same music will be delivered in audio-only form to the sound system controlled by an onboard computer. Such a scenario involving user mobility and application continuity is called application-level handoff.

M echanisms to support application-level handoff need to be incorporated into the Q OS setup protocol. First, the deployment of a user tracking system is necessary. I t keeps track of users, and maintains information about each user, including the user's ID, carry-on device(s), and current location. Second, the following additional steps are performed during Q oS setup for a mobile user:

- When the user arrives at a new location, the user tracking system is invoked to recognize the user, and to update and retrieve 


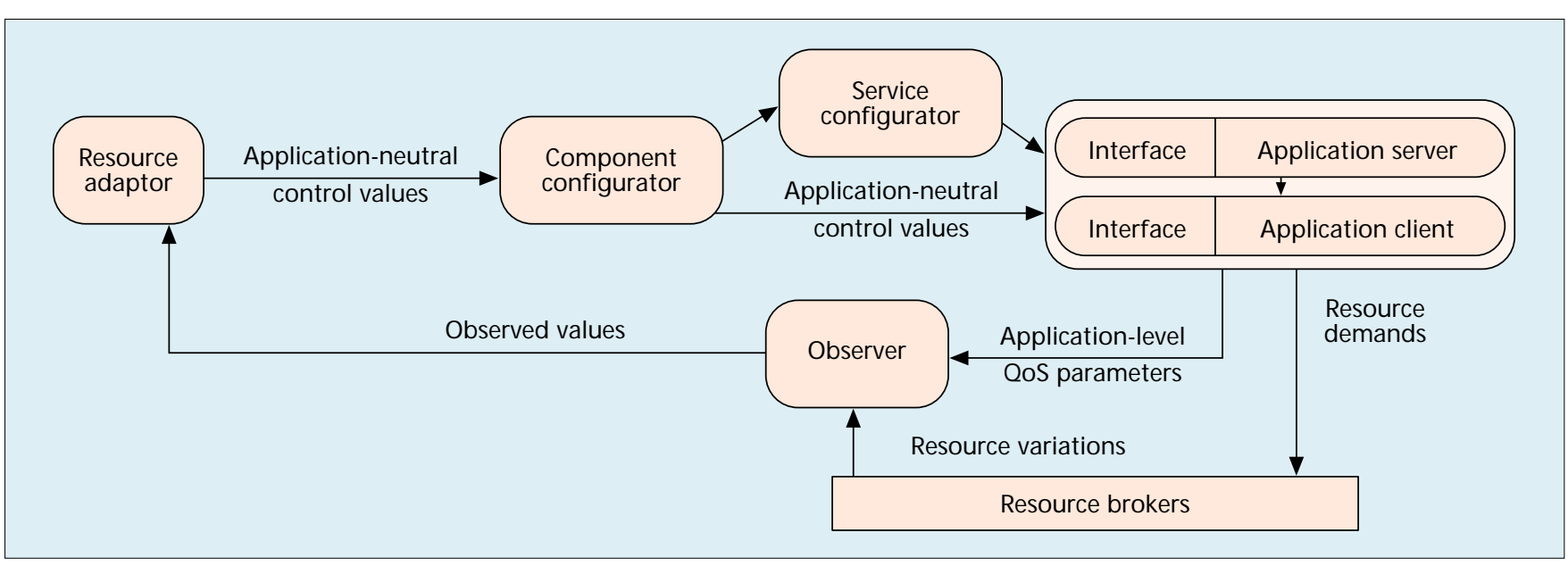

Figure 4. Viewing Q oS adaptation as a control loop.

the corresponding user information. Contact with the user tracking system can be either initiated manually by the user (e.g., via user logon) or triggered automatically by an active user detection device - for example, each user could carry an intelligent badge capable of automatic handshake with a computer, based on the computer's proximity to the user.

- A pausing application started earlier by this user does not have to be requested again. Instead, Q oS setup can resume its execution automatically. First, the usual steps of Q oS setup will be performed, including service discovery (which may be necessary due to the user's location change), application configuration (re)selection, and resource allocation.

- Then the intermediate execution state of the application will be retrieved and restored by the client-side Q oSProxy. The execution state can be retrieved either as part of the user information from the user tracking system, or from the user's carry-on device, one the user always carries with him/her (e.g., a PD A). H owever, this requires that the state be captured when the user moves away from the previous location. To do this, when the user moves away, the Q oSProxy of the previous client takes a "snapshot" of the application execution, according to the downloaded application state template (recall step 4 in the previous section). The snapshot, which contains necessary state information to properly resume the application, is then sent to either the user tracking system or the user's carryon device.

\section{Runtime Phase: QOS Adaptation}

A t runtime, after Q oS setup, the Q oS-aware middleware may perform Q oS adaptation during the execution of an application. R ecall that in an earlier section the end-to-end resource assignment for each application configuration is the minimum assignment, based on the lowest acceptable Q $\mathrm{OS}$ delivered by this configuration. Therefore, during the execution, the delivered application Q oS should be dynamically adjusted according to the actual resource availability. In a worse case, the environment might not even support resource reservations. In both cases, runtime $\mathrm{Q}$ oS adaptation is necessary.

Q OS adaptation takes place at both the resource management and service management levels. A t resource management level, R esource observers and adaptors perform application-neutral adaptation. A the service management level, Q oSProxies perform adaptation on application components and configurations based on application adaptation policies, a part of the Q oS profile (discussed earlier). A $n$ integrated model of all adaptations is shown in Fig. 4 as a control loop.

\section{QOS AdAPTATION BY RESOURCE ADAPTORS}

$R$ esource adaptors are neutral to applications and specific to resource types, such as CPU and network bandwidth. A resource adaptor controls all concurrent applications sharing the same resource in an end host. It reacts to resource fluctuation by fairly reallocating the available resource to the sharing applications and notifying them of the changes. E ach application, in turn, will adapt the rate, volume, or fidelity of its application data according to the resource allocation changes. Notice that in this type of Q OS adaptation, only application data are adapted, while the application configuration remains unchanged.

\section{QOS ADAPTATION BY COMPONENT CONFIGURATORS}

As part of the Q oSProxy, a component configurator performs Q oS adaptation at a higher level. This type of QOS adaptation involves the replacement, deletion, or addition of application component(s) in the application configuration. A ctions of component configurators are defined in the application adaptation policies (part of the Q oS profile). The policies are based on the fuzzy control model [2]. The adoption of fuzzy logic is justified by the observation that multiple reconfiguration and parameter-tuning options span different domains, and that the controllable regions and variables within the application are discrete and nonlinear. In such a scenario, fuzzy logic allows the specification of such a decisionmaking process with a small number of fuzzy 


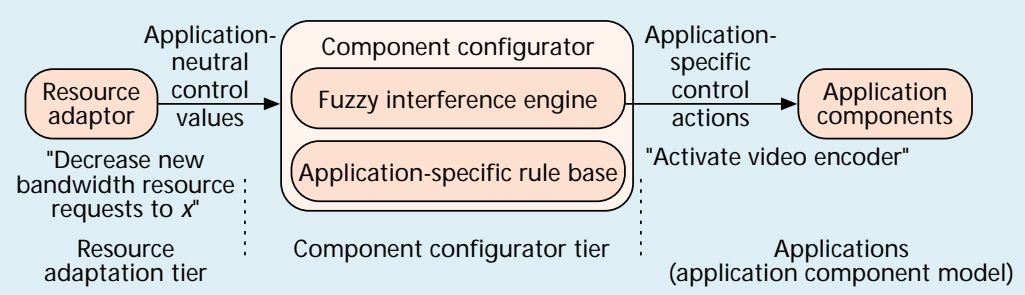

(a) QoS adaptation by component configurator

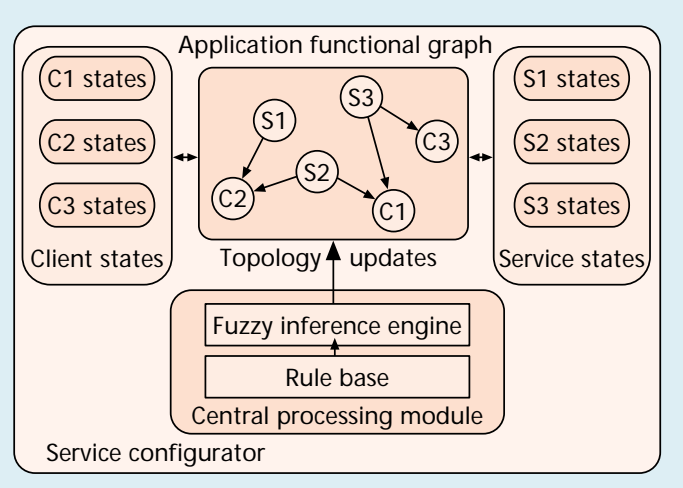

(b) QoS adaptation by service configurator

Figure 5. Q oS adaptation at the service management level.

rules. The nonlinearity of the fuzzy controller naturally matches the complexities brought by having multiple adaptation choices.

The fuzzy control model utilizes fuzzy logic to express application adaptation policies as a configurable rule base, which "fuels" a generic fuzzy inference engine to derive the exact control decisions. It contains two parts: linguistic rules consisting of a set of linguistic variables and values, and membership functions for linguistic values. $A$ typical linguistic rule is:

if (cpu is high) and (rate is low) then rateaction : = activate_encoder;

Such a rule specifies that if the CPU adaptor allocates $C P U$ in high amounts but the bandwidth adaptor allocates bandwidth at low rates, reconfigure the application to activate the video encoder application component. A typical membership function for a linguistic value such as high can be expressed with four deterministic points of any trapezoid-shaped membership functions, depending on adaptation requirements. The output of the function is in the range of $[0,1]$, representing the possibility that adaptation should happen.

The application-neutral output of the resource adaptors is piped into the component configurator, fuzzified as input to the inference engine based on its rule base. A ny output from the inference engine is then the Q OS adaptation decision for the application. For example, in Fig. $5 a$, the application-neutral output of the bandwidth adaptor may be decrease new bandwidth resource requests to $x$, and the output of the component configurator may be activate the video encoder H.261.

\section{QoS Adaptation by Service Configurators}

A s part of the Q oSProxy, the service configurator performs Q OS adaptation in an end-to-end fashion, removing the limitation that $\mathrm{Q} O S$ adaptation can only be performed in a single end host. M ore specifically, the service configurator is able to change the application configuration selected during the Q oS setup phase (see the previous section). For example, in 0 mnitrack, a distributed visual tracking application [2], when the end-toend bandwidth becomes unacceptably low, the service configurator will decide that the best Q oS adaptation is to switch to another video camera server with a low-bit-rate video codec.

Internally, the service configurator maintains a state table for each of the clients and servers, as well as an application functional graph representing the currently selected application configuration (as shown in Fig. 5b). If a reconfiguration occurs, the graph will be updated correspondingly. The central processing module makes the Q oS adaptation decisions. W e again adopt the fuzzy-logic-based fuzzy inference engine for the purpose of processing input states from hosts and generating an application reconfiguration decision. A s in the component configurator, such an inference process is also based on application adaptation policies (part of the Q OS profile) expressed as a rule base, and on states of individual hosts in the application functional graph. In the Omnitrack example, a rule in the rule base can be:

if (server_load is low) and (server_angle is close) then server_ranking is high;

In this example, server_angle is a dynamically generated value derived from the state of a particular client and server, including the actual angle of the client's desired view, the view that the server offers, and the difference between them. On the other hand, server_load is the observed CPU load on a server. The better a server matches this criteria, the higher the ranking of a server will have. The highest ranked server should be selected to serve the client; therefore, the application configuration involving this server will be selected and instantiated.

\section{A COMPARISON OF QOS-AWARE MIDDLEWARE SYSTEMS}

Table 1 provides a comparison of existing Q OSaware middleware systems: $2 \mathrm{KQ}$ [9], A gilos [2], Q oS services in CORBA, TA O [10], QuO [11], Q oSM E [1], H afid and B ochmann's Q oS management framework [12], and Q-RAM [3]. W e compare these systems in the following aspects: Q oS specification, Q oS translation, supported applications, Q OS enforcement, and Q OS adaptation. 


\begin{tabular}{|c|c|c|c|c|c|}
\hline $\begin{array}{l}\text { QoS middleware } \\
\text { system }\end{array}$ & QoS specification & QoS translation & $\begin{array}{l}\text { Range of supporting } \\
\text { applications }\end{array}$ & QoS enforcement & QoS adaptation \\
\hline $2 \mathrm{KQ}$ & $\begin{array}{l}\text { QoS specifications using } \\
\text { a QoS programming } \\
\text { environment }\end{array}$ & $\begin{array}{l}\text { Multiphase QoS } \\
\text { compilations }\end{array}$ & $\begin{array}{l}\text { Multiple domains of } \\
\text { applications via } \\
\text { customizable QoS } \\
\text { specifications and multi- } \\
\text { phase compilations }\end{array}$ & $\begin{array}{l}\text { Guaranteeing } \\
\text { minimum-amount } \\
\text { reservation of } \\
\text { resources }\end{array}$ & $\begin{array}{l}\text { Intraconfiguration } \\
\text { adaptation and } \\
\text { dynamic } \\
\text { reconfiguration }\end{array}$ \\
\hline $\begin{array}{l}\text { QoS in CORBA audio/ } \\
\text { video streaming } \\
\text { service }\end{array}$ & $\begin{array}{l}\text { Predefined IDL } \\
\text { interfaces extending the } \\
\text { standard CORBA IDL } \\
\text { interfaces }\end{array}$ & Internal translation & $\begin{array}{l}\text { Audio/video streaming } \\
\text { applications }\end{array}$ & $\begin{array}{l}\text { Performed by } \\
\text { available } \\
\text { transport protocols }\end{array}$ & $\begin{array}{l}\text { Adaptation at } \\
\text { network } \\
\text { transport layer }\end{array}$ \\
\hline Real-time CORBA & $\begin{array}{l}\text { Predefined IDL } \\
\text { interfaces extending the } \\
\text { standard CORBA IDL } \\
\text { interfaces }\end{array}$ & N/A & Real-time applications & $\begin{array}{l}\text { Performed by } \\
\text { real-time extensions } \\
\text { in standard OS }\end{array}$ & N/A \\
\hline Fault-tolerant CORBA & $\begin{array}{l}\text { Predefined IDL } \\
\text { interfaces extending the } \\
\text { standard CORBA IDL } \\
\text { interfaces }\end{array}$ & N/A & Fault-tolerant applications & $\begin{array}{l}\text { Maintaining } x \\
\text { replicas for a certain } \\
\text { fault-tolerant level }\end{array}$ & N/A \\
\hline TAO & $\begin{array}{l}\text { Predefined IDL } \\
\text { interfaces }\end{array}$ & $\mathrm{N} / \mathrm{A}$ & $\begin{array}{l}\text { Real-time messaging } \\
\text { applications }\end{array}$ & $\begin{array}{l}\text { Based on priority } \\
\text { queues in ORB }\end{array}$ & $N / A$ \\
\hline QoSME & $\begin{array}{l}\text { Quality Assurance } \\
\text { Language (QuAL) }\end{array}$ & $\mathrm{N} / \mathrm{A}$ & $\begin{array}{l}\text { Applications requiring } \\
\text { QoS in transport protocols } \\
\text { and in OS }\end{array}$ & $\begin{array}{l}\text { Performed by } \\
\text { available } \\
\text { transport protocols } \\
\text { and POSIX- } \\
\text { compliant OS }\end{array}$ & $\begin{array}{l}\text { Using a set of } \\
\text { predefined } \\
\text { operators for } \\
\text { QoS renegotiation }\end{array}$ \\
\hline Q-RAM & $\begin{array}{l}\text { Resource utility } \\
\text { functions }\end{array}$ & $\mathrm{N} / \mathrm{A}$ & $\begin{array}{l}\text { Applications running in } \\
\text { a resource-sharing } \\
\text { environment and } \\
\text { requiring QoS provision }\end{array}$ & $\begin{array}{l}\text { Based on their } \\
\text { resource allocation } \\
\text { model }\end{array}$ & $\begin{array}{l}\text { By their adaptive } \\
\text { resource allocation } \\
\text { algorithms }\end{array}$ \\
\hline
\end{tabular}

Table 1. Comparison of Q OS-aware middleware systems.

\section{CONCLUSION}

Q oS-aware middleware systems have emerged to assist a new spectrum of applications that require Q OS in heterogenous and ubiquitous computing environments. In this article we have shown that, using an application component model, it is possible to provide end-to-end application Q OS via Q oS-aware middleware systems, by:

- Generating appropriate Q oS specifications

- Translating and compiling multiple application configurations for the same application to be run in heterogeneous environments

- Selecting an appropriate configuration and discovering the participating application components

- A dapting Q oS at multiple levels and with different granularities in case of Q oS degradations.

Our own experiences with QoS-aware middleware systems, such as $2 \mathrm{~K}^{\mathrm{Q}}$ and A gilos, provided us with several lessons. First, it is difficult to design a uniform Q OS specification language to allow for Q OS description in different applica- 


The results from
our current
development of
QoS-aware
middleware
systems are
encouraging. We
believe that such
middleware will
become an
integral
constituent of
the application-
enabling platform
for emerging
ubiquitous and
heterogeneous
environments.

tion domains, and further research is needed. Second, Q oS compilations may require application code instrumentation, and hence developer awareness, because not all translations from application Q oS to resource assignment can be automated. Third, the resource-level Q oS support via resource brokers, such as CPU and bandwidth brokers, are highly desirable for the provision of end-to-end application Q OS. A middleware system can deliver much better end-toend Q OS if it collaborates with the underlying OS and network Q oS support. F inally, Q oS adaptation capability is necessary in middleware systems, especially if they are to assist applications on top of best effort os and networks.

$O$ verall, the results from our current development of Q oS-aware middleware systems are encouraging. We believe that such middleware will become an integral constituent of the application-enabling platform for emerging ubiquitous and heterogeneous environments.

\section{ACKNOWLEDGMENTS}

We would like to thank the anonymous reviewers for their valuable comments on this article. This work was supported by the A ir Force under contract F 30602-97-2-0121, ONR MURI under contract NA VY CU 37515-6281, and the National Science Foundation under contracts N SF CCR 0086094, NSF EIA 9972884, and NSF EIA 9870736 .

\section{REFERENCES}

[1] P. G. S. Florissi, “QoSME: QoS Management Environment," Ph.D. thesis, Columbia Univ., 1996.

[2] B. Li and K. Nahrstedt, "A Control-Based Middleware Framework for Quality of Service Adaptation," IEEE JSAC, Special Issue on Service Enabling Platforms, vol. 17., no. 9, Sept. 1999, pp. 1632-50.

[3] R. Rajkumar et al., "A Resource Allocation Model for QoS Management," IEEE Real-Time Sys. Symp., Dec. 1997, pp. 298-307.

[4] D. Wichadakul and K. Nahrstedt, "Distributed OoS Compiler," Technical Report UIUCDCS-R-2001-2001, Dept. Comp. Sci., Univ. of IL at Urbana-Champaign, submitted for journal publication, Feb. 2001.

[5] E. Guttman, "Service Location Protocol: Automatic Discovery of IP Network Services," IEEE Internet Comp., vol. 3, no. 4, 1999, pp. 71-80.

[6] J. Waldo, "The Jinni Architecture for Network-Centric Computing," Commun. ACM, vol. 42, no. 7, July 1999. pp. 76-82.

[7] S. Czerwinski et al., "An Architecture for a Secure Discovery Service," ACM Mobicom, Sept. 1999, pp. 24-35.

[8] D. Xu, K. Nahrstedt, and D. Wichadakul, "QoS-Aware Discovery of Wide-Area Distributed Services," Proc. IEEE/ACM Int'l. Symp. Cluster Comp. and Grid, CCGrid '01, May 2001, pp. 92-99.

[9] K. Nahrstedt, D. Wichadakul, and D. Xu, "Distributed QoS Compilation and Runtime Instantiation," Proc. 8th IEEE/IFIP Int'I. Wksp. QoS, June 2000, pp. 198-207.

[10] D. Schmidt, D. Levine, and C. Cleeland, "Architectures and Patters for High-Performance, Real-Time CORBA Object Request Brokers," Advances in Comp., Marvin Zelkowitz, Ed., Academic Press, 1999.

[11] J. Znky, D. Bakken, and R. Schantz, "Architecture Support for Quality of Service for CORBA Objects," Theory and Practice of Object Sys., vol. 3, no. 1, Jan. 1997.

[12] A. Hafid and G. Bochman, "An Approach to OoS Management in Distributed Multimedia Applications: Design and Implementation," Multimedia Tools and Applications, vol. 9, no. 2, 1999.

\section{BIOGRAPHIES}

KLARA NAHRSTEDT (klara@cs.uiuc.edu) [M] is an associate professor at the University of Illinois at Urbana-Champaign, Computer Science Department. Her research interests are directed toward reconfigurable multimedia services, multimedia protocols, multimedia security, middleware systems, Quality of Service (QoS) provision, QoS routing, and QoSaware resource management in distributed multimedia systems. She is the co-author of the widely used multimedia book Multimedia: Computing, Communications and Applications published by Prentice Hall, the recipient of the Early NSF Career Award, the Junior Xerox Award, and IEEE Communication Society Leonard Abraham Award for Research Achievements. Since June 2001 she serves as editor-in-chief of ACM Multimedia Systems Journal. She received her B.A. in mathematics from Humboldt University, Berlin, Germany, in 1984, and her M.Sc. degree in numerical analysis from the same university in 1985 . She was a research scientist in the Institute for Informatik in Berlin until 1990. In 1995 she received her Ph.D. from the University of Pennsylvania in the Department of Computer and Information Science. She is member of ACM and SPIE.

DONGYAN XU (d-Xu@cs.uiuc.edu) [StM] received his B.S. in computer science from Zhongshan University, China. He is currently a Ph.D. candidate in the Department of Computer Science at the University of Illinois at Urbana-Champaign His research interests include QoS in distributed multimedia systems, mobile computing and networking, and Internet computing. He is a student member of ACM.

DUANGDAO WICHADAKUL (wichadak@cs.uiuc.edu) received her B.E. in computer engineering from Chulalongkorn University, Thailand, and her M.S. in computer science from the University of Illinois at Urbana-Champaign. Currently she is a Ph.D. candidate in the Department of Computer Science at the University of Illinois at Urbana-Champaign. Her research interests include QoS in distributed object computing and ubiquitous environments, QoS specification and compilation, monitoring, and probing techniques.

BAOCHUN L (bli@eecg.toronto.edu) received his B.E. in computer science from Tsinghua University, China, and his M.S and Ph.D. degrees in computer science from the University of Illinois at Urbana-Champaign. Currently he is an assistant professor at the Department of Electrical and Computer Engineering of the University of Toronto. His research interests include QoS, application-level monitoring and adaptation, multimedia, and mobile computing. 